\title{
Generation of bone marrow chimeras using X-ray irradiation: comparison to cesium irradiation and use in immunotherapy
}

Jason Eng, Jessica Orf, Kristy Perez, Deepali Sawant, Jason DeVoss*

Amgen Research, Department of Oncology, South San Francisco, CA 94080, USA

Figure S1 - Irradiation and reconstitution results in a delay in MC38 tumor growth
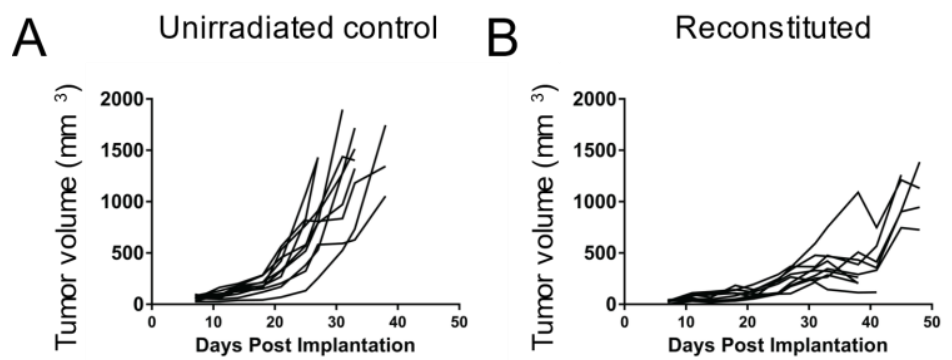

Figure S1 - Irradiation and reconstitution results in a delay in MC38 tumor growth. Age matched unirradiated controls (A) or $7 \mathrm{~Gy}$ irradiated and reconstituted (B) C57BI6 animals were implanted with 3e5 MC38 syngeneic colorectal tumor cells. This figure is representative of at least two independent experiments. 
Figure S2 - Gating schemes for identification of populations by flow cytometry

A

Lymphocyte and DC gating strategy
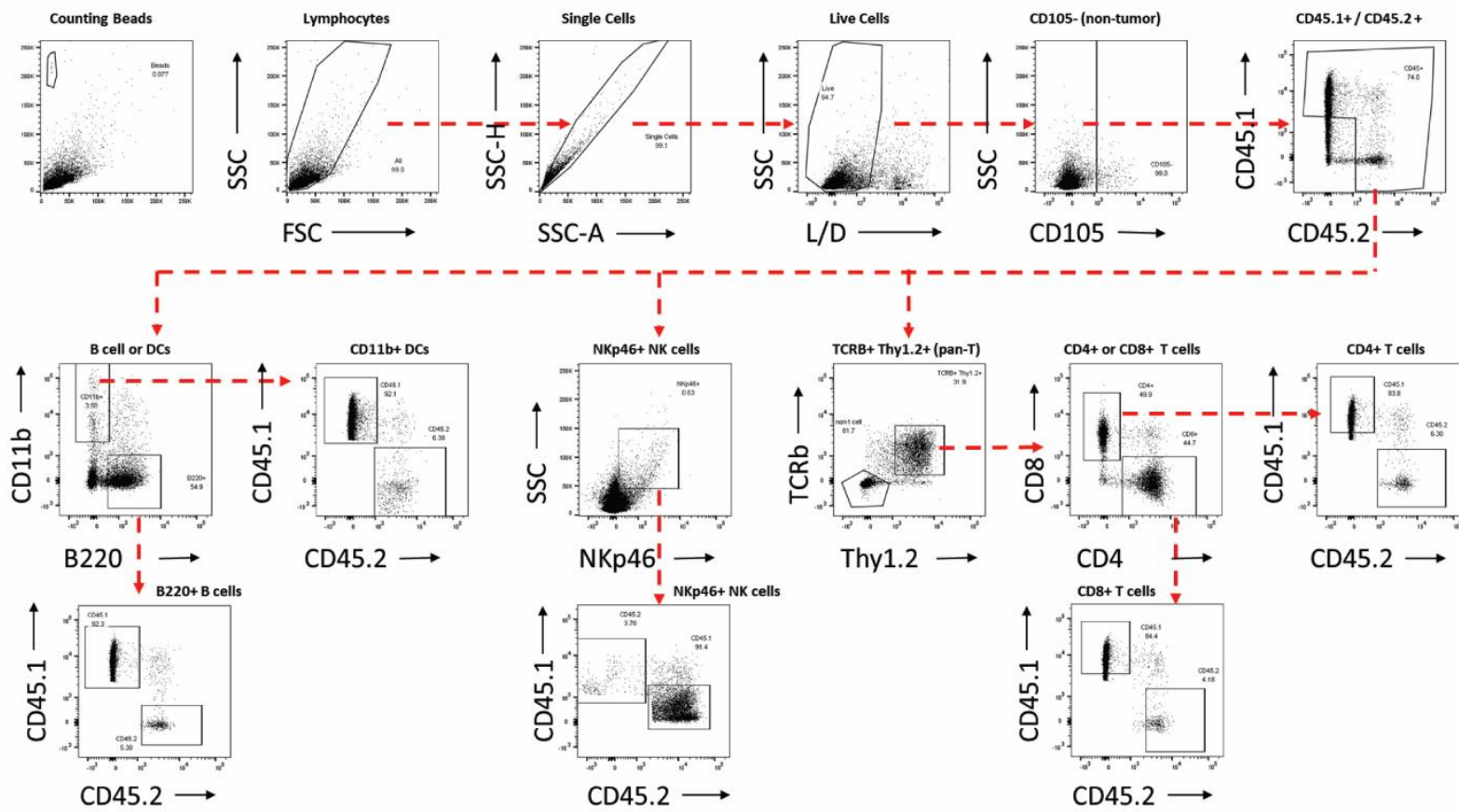

Thy1.2 $\longrightarrow$

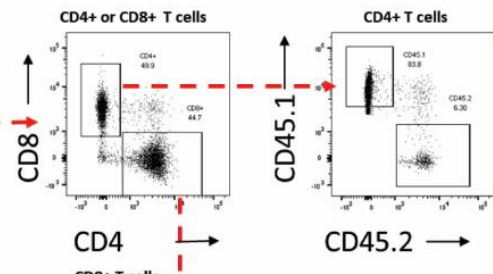

$\mathrm{CD} 45.2 \longrightarrow$

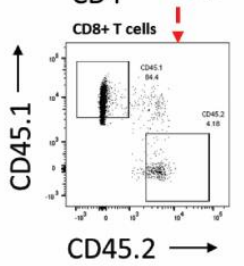

B Myeloid and neutrophil gating
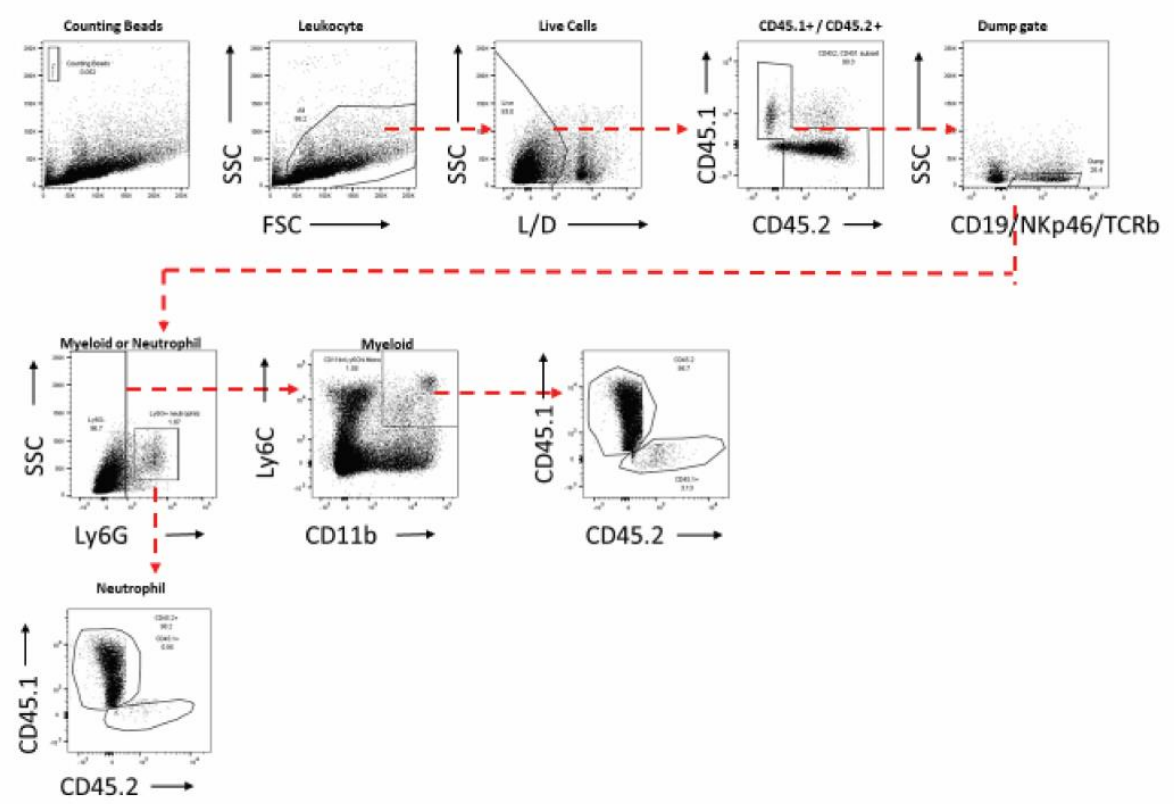

Figure S2 - Gating schemes for identification of populations by flow cytometry. This is representative of the lymphoid (top) and myeloid (bottom) gating strategies used throughout the paper. 
Figure S3 - Confirmation of chimerism in blood and spleen of tumor engrafted animals
A
Blood 7 gy X-ray
B Blood 9 gy Cesium
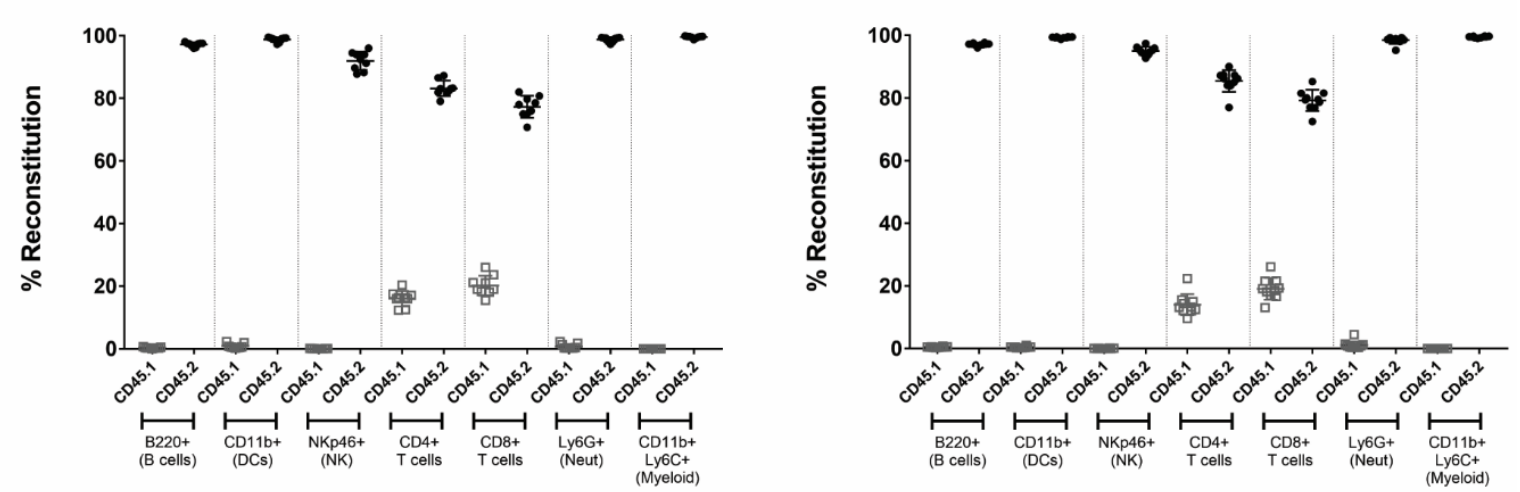

C Spleen 7 gy X-ray

D Spleen Cesium 9 gy
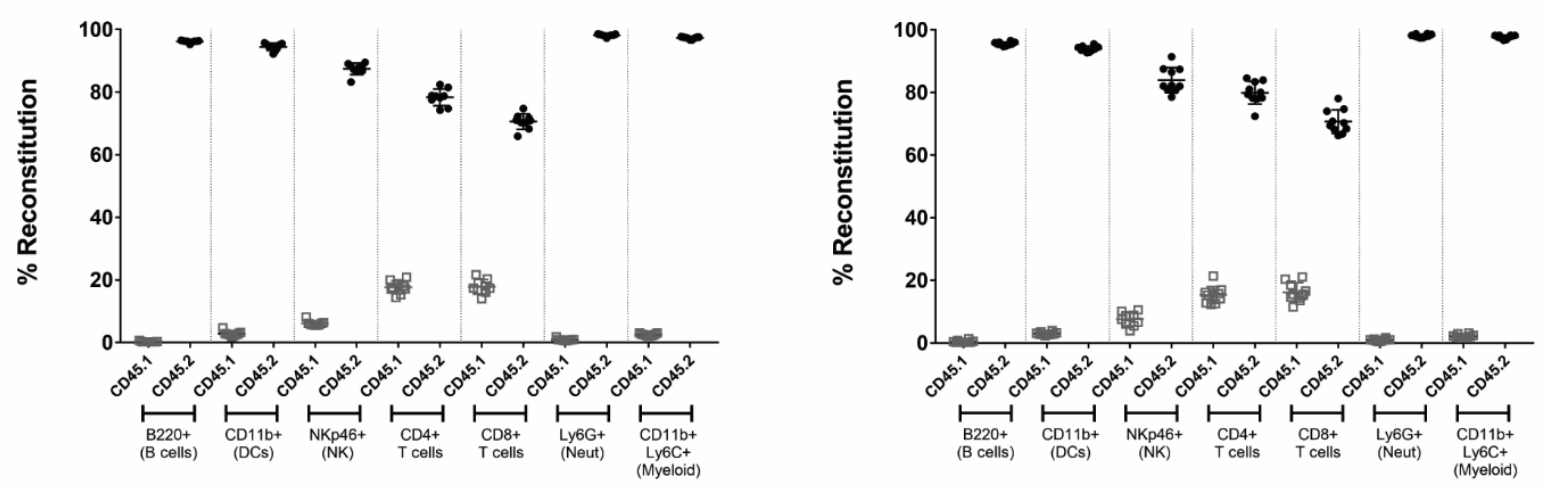

Figure S3 - Confirmation of chimerism in blood and spleen of tumor engrafted animals. Donor marrow reconstitution was evaluated in animals that were irradiated with X-ray ( $\mathbf{A}$ and $\mathbf{C}$ ) or Cesium ${ }^{137}$ (B and $\left.\mathbf{D}\right)$. Donor hematopoietic cells (CD45. $2^{+}$; closed circles) were differentiated from recipient hematopoietic cells (CD45.1 $1^{+}$; open squares) by flow cytometry using congenic markers. Hematopoietic cells were identified based on commonly expressed leukocyte antigens. The figure is representative of at least two independent experiments. 